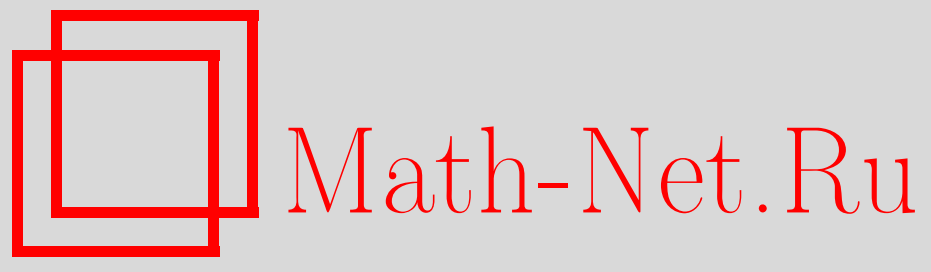

П. А. Крутицкий, Н. Ч. Крутицкая, О потенциалах статической теории упругости, УМH, 2009, том 64, выпуск 1, 149-150

DOI: https://doi.org/10.4213/rm9273

Использование Общероссийского математического портала Math-Net.Ru подразумевает, что вы прочитали и согласны с пользовательским соглашением http://www . mathnet.ru/rus/agreement

Параметры загрузки:

IP : 54.92 .164 .108

26 апреля 2023 г., 12:44:41

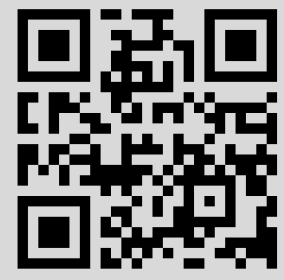




\section{О потенциалах статической теории упругости}

\section{П. А. Крутицкий, Н. Ч. Крутицкая}

Под разомкнутой кривой понимается простая гладкая незамкнутая дуга конечной длины без самопересечений. В декартовых координатах $x=\left(x_{1}, x_{2}\right) \in \mathbb{R}^{2}$ на плоскости рассмотрим простую гладкую разомкнутую кривую $\Gamma$ класса $\mathbb{C}^{1, \lambda}, \lambda \in(0,1]$, параметризованную длиной дуги $s: \Gamma=\left\{x: x=x(s)=\left(x_{1}(s), x_{2}(s)\right), s \in[a, b]\right\}$. Тем самым, между точками $x \in \Gamma$ и значениями параметра $s$ имеется взаимно однозначное соответствие. Вектор касательной к кривой $\Gamma$ в точке $x(s)$, указывающий направление возрастания параметра $s$, обозначим $\tau_{x}=(\cos \alpha(s), \sin \alpha(s))$, а вектор нормали, совпадающий с вектором касательной при повороте на угол $\pi / 2$ против часовой стрелки, обозначим $\mathbf{n}_{x}=(\sin \alpha(s),-\cos \alpha(s))$. При выбранной параметризации $\cos \alpha(s)=x_{1}^{\prime}(s), \sin \alpha(s)=x_{2}^{\prime}(s)$. Отрезок $[a, b]$ будет также обозначаться $\Gamma$.

Пусть $\mathbb{C}^{0, \omega}[a, b]$ - класс функций, гёльдеровых с показателем $\omega$ на $[a, b]$. Будем говорить, что $\mathscr{F}(s) \in \mathbb{C}_{q}^{\omega}[a, b]$, если $\mathscr{F}_{0}(s) \in \mathbb{C}^{0, \omega}[a, b]$, где $\mathscr{F}_{0}(s)=\mathscr{F}(s)|s-a|^{q}|s-b|^{q}$.

Рассмотрим $\Gamma$ как разрез на плоскости. Ту сторону разреза $\Gamma$, которая остается слева при возрастании параметра $s$, обозначим $\Gamma^{+}$, а противоположную сторону обозначим $\Gamma^{-}$.

Векторное уравнение статической теории упругости в координатах $\left(x_{1}, x_{2}\right)$ относительно вектора $\mathbf{v}=\left(v_{1}, v_{2}\right)$ имеет вид $\eta \Delta \mathbf{v}+(\kappa+\eta) \operatorname{grad} \operatorname{div} \mathbf{v}=\mathbf{0}$, где $\eta, \kappa$-константы такие, что $\eta \neq 0, \kappa \neq-2 \eta$ и $\kappa \neq-3 \eta$.

Пусть $\mu_{1}(s), \mu_{2}(s)$ - интегрируемые функции, заданные на $[a, b]$. Введем столбец $\mu(s)=\left(\mu_{1}(s), \mu_{2}(s)\right)^{T}$. Цель настоящей заметки - изучить потенциалы статической теории упругости, которые можно записать в виде:

$$
\begin{aligned}
V_{1}[\mu](x)=\frac{p_{1}}{4 \pi} & \left\{-\int_{\Gamma} \mu_{1}(\sigma) \ln |x-y(\sigma)| d \sigma+p_{2} \int_{\Gamma} \mu_{1}(\sigma) \frac{1+\cos (2 \psi(x, y(\sigma)))}{2} d \sigma\right. \\
& \left.+p_{2} \int_{\Gamma} \mu_{2}(\sigma) \frac{\sin (2 \psi(x, y(\sigma)))}{2} d \sigma\right\}, \\
V_{2}[\mu](x)= & \frac{p_{1}}{4 \pi}\left\{p_{2} \int_{\Gamma} \mu_{1}(\sigma) \frac{\sin (2 \psi(x, y(\sigma)))}{2} d \sigma-\int_{\Gamma} \mu_{2}(\sigma) \ln |x-y(\sigma)| d \sigma\right. \\
& \left.+p_{2} \int_{\Gamma} \mu_{2}(\sigma) \frac{1-\cos (2 \psi(x, y(\sigma)))}{2} d \sigma\right\},
\end{aligned}
$$

где $p_{1}=\frac{\kappa+3 \eta}{\eta(\kappa+2 \eta)}, p_{2}=\frac{\kappa+\eta}{\kappa+3 \eta}, \cos \psi(x, y(\sigma))=\frac{x_{1}-y_{1}(\sigma)}{|x-y(\sigma)|}, \sin \psi(x, y(\sigma))=\frac{x_{2}-y_{2}(\sigma)}{|x-y(\sigma)|}$,

$$
|x-y(\sigma)|=\sqrt{\left(x_{1}-y_{1}(\sigma)\right)^{2}+\left(x_{2}-y_{2}(\sigma)\right)^{2}}, \quad y(\sigma)=\left(y_{1}(\sigma), y_{2}(\sigma)\right) \in \Gamma,
$$

и использованы формулы $\cos (2 \psi)=2 \cos ^{2}(\psi)-1=1-2 \sin ^{2}(\psi), \sin (2 \psi)=2 \sin \psi \cos \psi$.

Теорема. Пусть Г - разомкнутая кривая класса $\mathbb{C}^{1, \lambda}, \lambda \in(0,1]$. Кроме того, пусть $\mu_{1}(\sigma), \mu_{2}(\sigma) \in \mathbb{C}_{q}^{\omega}[a, b], \omega \in(0,1], q \in[0,1)$. Тогда:

1) функиии $V_{1}[\mu](x), V_{2}[\mu](x)$ принадлежат $\mathbb{C}^{0}\left(\mathbb{R}^{2}\right) \cap \mathbb{C}^{2}\left(\mathbb{R}^{2} \backslash \Gamma\right)$, а $\nabla V_{1}[\mu](x)$ u $\nabla V_{2}[\mu](x)$ принадлежат ${ }^{1} \mathbb{C}^{0}\left(\overline{\mathbb{R}^{2} \backslash \Gamma} \backslash X\right)$, где $X=x(a) \cup x(b)-$ множество концов $\Gamma$;

Работа выполнена при поддержке РФФИ (гранты № 08-01-00082, 09-01-00025).

${ }^{1}$ Через $\mathbb{C}^{0}\left(\overline{\mathbb{R}^{2} \backslash \Gamma} \backslash X\right)$ обозначается класс функций, которые непрерывны вне $\Gamma$, непрерывно продолжимы на стороны разреза Г слева и справа во внутренних точках, но предельные значения каждой функции на $Г$ слева и справа могут быть разными. 
2) предельные значения нормальной и касательной производных потенииалов $V_{1}[\mu](x)$ и $V_{2}[\mu](x)$ на $\Gamma^{ \pm} \backslash X$ даются формулами

$$
\begin{aligned}
& \left.\frac{\partial V_{1}[\mu](x)}{\partial \tau_{x}}\right|_{x=x(s) \in \Gamma^{ \pm}}=\frac{\partial V_{1}[\mu](x(s))}{\partial s}=\frac{p_{1}}{4 \pi}\left\{-\int_{\Gamma} \mu_{1}(\sigma) \frac{\cos (\psi(x, y(\sigma))-\alpha(s))}{|x-y(\sigma)|} d \sigma\right. \\
& \quad+p_{2} \int_{\Gamma} \mu_{1}(\sigma) \sin (2 \psi(x, y(\sigma))) \frac{\sin (\psi(x, y(\sigma))-\alpha(s))}{|x-y(\sigma)|} d \sigma \\
& \left.\quad-p_{2} \int_{\Gamma} \mu_{2}(\sigma) \cos (2 \psi(x, y(\sigma))) \frac{\sin (\psi(x, y(\sigma))-\alpha(s))}{|x-y(\sigma)|} d \sigma\right\}, \\
& \left.\frac{\partial V_{2}[\mu](x)}{\partial \tau_{x}}\right|_{x=x(s) \in \Gamma^{ \pm}}=\frac{\partial V_{2}[\mu](x(s))}{\partial s}=\frac{p_{1}}{4 \pi}\left\{-\int_{\Gamma} \mu_{2}(\sigma) \frac{\cos (\psi(x, y(\sigma))-\alpha(s))}{|x-y(\sigma)|} d \sigma\right. \\
& \quad-p_{2} \int_{\Gamma} \mu_{1}(\sigma) \cos (2 \psi(x, y(\sigma))) \frac{\sin (\psi(x, y(\sigma))-\alpha(s))}{|x-y(\sigma)|} d \sigma \\
& \left.\quad-p_{2} \int_{\Gamma} \mu_{2}(\sigma) \sin (2 \psi(x, y(\sigma))) \frac{\sin (\psi(x, y(\sigma))-\alpha(s))}{|x-y(\sigma)|} d \sigma\right\}, \\
& \left.\frac{\partial V_{1}[\mu](x)}{\partial \mathbf{n}_{x}}\right|_{x=x(s) \in \Gamma^{ \pm}}=\frac{p_{1}}{4 \pi}\left\{\int_{\Gamma} \mu_{1}(\sigma) \frac{\sin (\psi(x, y(\sigma))-\alpha(s))}{|x-y(\sigma)|} d \sigma\right. \\
& \quad+p_{2} \int_{\Gamma} \mu_{1}(\sigma) \sin (2 \psi(x, y(\sigma))) \frac{\cos (\psi(x, y(\sigma))-\alpha(s))}{|x-y(\sigma)|} d \sigma \pm \pi p_{2} \mu_{2}(s) \sin (2 \alpha(s)) \\
& \left.\quad-p_{2} \int_{\Gamma} \mu_{2}(\sigma) \cos (2 \psi(x, y(\sigma))) \frac{\cos (\psi(x, y(\sigma))-\alpha(s))}{|x-y(\sigma)|} d \sigma \pm \pi \mu_{1}(s)\left(1+p_{2} \cos (2 \alpha(s))\right)\right\} \\
& \left.\frac{\partial V_{2}[\mu](x)}{\partial \mathbf{n}_{x}}\right|_{x=x(s) \in \Gamma^{ \pm}}=\frac{p_{1}}{4 \pi}\left\{ \pm \pi p_{2} \mu_{1}(s) \sin (2 \alpha(s))+\int_{\Gamma} \mu_{2}(\sigma) \frac{\sin (\psi(x, y(\sigma))-\alpha(s))}{|x-y(\sigma)|} d \sigma\right. \\
& -p_{2} \int_{\Gamma} \mu_{1}(\sigma) \cos (2 \psi(x, y(\sigma))) \frac{\cos (\psi(x, y(\sigma))-\alpha(s))}{|x-y(\sigma)|} d \sigma \pm \pi \mu_{2}(s)\left(1-p_{2} \cos (2 \alpha(s))\right) \\
& \left.\quad p_{2} \mu_{2}(\sigma) \sin (2 \psi(x, y(\sigma))) \frac{\cos (\psi(x, y(\sigma))-\alpha(s))}{|x-y(\sigma)|} d \sigma\right\},
\end{aligned}
$$

где интеграль, содержащие $\cos (\psi(x, y(\sigma))-\alpha(s))$, понимаются в смысле главного значения;

3) для любой точки $x \notin \Gamma$, расположенной в малой окрестности конца $x(d)(d=a$ или $d=b)$, выполняются неравенства

$$
\left|\frac{\partial V_{1}[\mu](x)}{\partial x_{j}}\right| \leqslant \frac{\text { const }}{|x-x(d)|^{\delta}}, \quad\left|\frac{\partial V_{2}[\mu](x)}{\partial x_{j}}\right| \leqslant \frac{\text { const }}{|x-x(d)|^{\delta}}, \quad j=1,2,
$$

где $\delta=q$, если $q \in(0,1)$, и $\delta$ - произвольное число из интервала $(0,1)$, если $q=0$.

Теорема вытекает из результатов работ [1], [2].

\section{Список литературы}

[1] П. А. Крутицкий, Ж ЖВМ и МФ, 34:8-9 (1994), 1237-1257; англ. пер.: P. A. Krutitskii, Comput. Math. Math. Phys., 34:8-9 (1994), 1073-1090. [2] P. A. Krutitskii, Quart. Appl. Math., 65:3 (2007), 549-569.

\section{П. А. Крутицкий (Р. A. Krutitskii)}

Московский государственный университет им. М. В. Ломоносова

Н. Ч. Крутицкая (N. Ch. Krutitskaya) Московский государственный университет им. М. В. Ломоносова
Представлено М. И. Зеликиным Принято редколлегией 07.12 .2008 